\title{
Classical geometric illusion effects with nonclassical stimuli: Angular induction from decomposing lines into point arrays
}

\author{
ERNEST GREENE and JÓZSEF FISER \\ University of Southern California, Los Angeles, California
}

\begin{abstract}
Angular induction is the process by which one line segment can bias judgment of orientation and/or collinearity of another segment, and it has been established that the magnitude of error is a determinate function of the relative angle between the two. We examined how these known relationships are affected by decomposing the induction segment into an array of scattered points. The bias that was produced by such arrays was found to be consistent with a formal model of angular induction, with the strength of the effect decreasing as the scatter among the points was increased. This decline in strength was almost linear with a logarithmic transform of the dimensions of the stimulus array. We also evaluated the hypothesis that the induction stimulus is detected by one or more channels-for example, neurons-for which the sensitivity profiles are modeled as Gabor wavelets. The change in induction strength with increasing point scatter was not predicted by a single width of channel. However, the combined activity of an ensemble of channels that differed in width did match the perceptual effects if one also stipulated that each channel would respond maximally to a fine-line stimulus.
\end{abstract}

It is remarkable how easily an artist or draftsman can elicit recognition of visual objects by the use of fine lines. The lines effectively capture the transitions that distinguish an object from its background, or convey an impression of the surface gradients. Given the salience and flexibility of this stimulus, it is easy to understand how most discussions of perceptual mechanism assume line detection as the beginning point for the analysis of images (Gibson, 1950; Helmholtz, 1909/1962; Titchener, 1915). This strategy has been greatly reinforced by the discovery that neurons in primary visual cortex respond vigorously to line segments with a particular orientation (Hubel \& Wiesel, 1962) and has led to explanations of biological mechanisms in terms of the "trigger features," which are provided by the stimulus.

Nonetheless, it is clear that the nervous system can process a broad range of stimulus material other than line drawings; thus, the response to lines must be considered as part of a more general encoding strategy (Barlow, 1961, 1981). With respect to receptive fields and perception alike, much of the work in recent years has been to develop and test quantitative models that have the capacity to encode lines, edges, texture gradients, and other image attributes. An especially promising development has

\footnotetext{
We would like to thank Russell DeValois for his insightful comments, and Brian Nelson for testing of subjects. This research was supported in part by the Neuropsychology Foundation and by OTKA Grant 285-0813. J.F. is in the Department of Computer Science at USC. E.G. is also affiliated with the Neuropsychology Foundation, Los Angeles. Address correspondence to E. Greene, University of Southern California, Department of Psychology, Los Angeles, CA 90089-1061.
}

been the reconciliation of spatial position and spatial frequency issues by modeling receptive and perceptive field properties as Gabor functions (see Field, 1987, for an overview).

It should be recognized, however, that the testing of quantitative models has focused almost exclusively on the discrimination thresholds for blurred lines and edges. Could these methods be applied to other kinds of stimuli, such as a scattered array of points? Further, can the models be extended to describe other perceptual interactions, such as the distortions of judgment that create geometric illusions? To be specific, it is intuitively obvious that an array of scattered points has some similarity to a line segment, in that one can compare the two in terms of orientation and straightness. Yet beyond these general attributes, one does not have a method for scaling the degree to which these two types of stimuli are perceptually comparable.

It is possible that the perceptual effect of segments and scattered points can be compared by measuring their relative strength in biasing judgment of collinearity, a process that we describe as angular induction. One of the simplest methods for measuring this induction effect is to place an oblique test segment upon the page and require that a subject select a point in open space that appears to be aligned with it. We will describe the selection of the point as being a collinearity judgment, though it should be clear that the process has an operant component.

The collinearity judgment is normally fairly accurate when the test segment is presented alone, but the inclusion of a second "induction" segment will bias the selection of the point. It has been found (Greene, 1993a, 
$1993 \mathrm{~b}, 1994)$ that the size of the angular error $(\Psi)$ is a determinate function of the relative angle $(\theta)$ between the test segment and the induction segment.

We conducted two experiments. The goal of Experiment 1 was to determine whether stimuli that do not belong to the classical set of sharp or blurred lines can generate the effects of angular induction. Thus, for Experiment 1 , we constructed a set of elongated arrays with a specified degree of scatter of the points. Each member of the set was presented in a configuration that was appropriate for producing angular induction; the axis of the array was varied and the induction bias was measured by having a subject judge the collinearity of the test segment. The results indicated that the arrays produce induction effects that were comparable to the influence of straight line segments - differing in strength, but otherwise following the angular rule with respect to theta.

Our plan was to evaluate the data from the first experiment in a model that relates to receptive field properties of orientation-selective neurons. However, for the most part, the model has been derived by the use of $s p a$ tial frequency gratings, in which the brightness of stimulus regions varies as a smooth sinusoidal wave. Before applying such a model, it was important to resolve two questions that were not answered in Experiment 1. (1) Are the induction effects unique to the point-scatter stimulus, or can they be produced by a "blurred-line" stimulus? (2) Is the strength of the effect partly determined by the fact that the test segment has no scatter? These issues are elaborated in the following text; at this point, it is sufficient to say that the results from Experiment 2 answered these questions and encouraged the application of the "neural model" to the data from Experiment 1.

We adopt a neural model that assumes that these stimuli are encoded by the nervous system, perhaps as early as the primary visual cortex. Substantial evidence shows that the receptive fields in striate cortex have excitatory and inhibitory subfields that can be described by a Gabor function (Field \& Tolhurst, 1986; Jones \& Palmer, 1987a; Kulikowski \& Bishop, 1981a; Marcelja, 1980; Sakitt \& Barlow, 1982). Therefore, using a set of Gabor functions that differ in their spatial dimensions, one can evaluate the influence of the point arrays to predict the level of response that should be produced by each. Using our model, we tested whether the responses predicted by a simple Gabor-function model would correspond to the psychological data.

\section{EXPERIMENT 1}

\section{Method}

Stimuli. The lines and points of the stimulus pages were plotted on $8.5 \times 11$ in. paper with an Apple LaserWriter. The stimulus configuration generally consisted of the test segment whose collinearity was to be judged and an array of induction points (called the induction array) that formed either a line or a scattered band. An example of the basic configuration is shown in Figure 1. The dimensions of and among image elements were measured with a dissecting microscope, which contained a calibrated reticule. These are reported as visual angles, in degrees and minutes of arc.

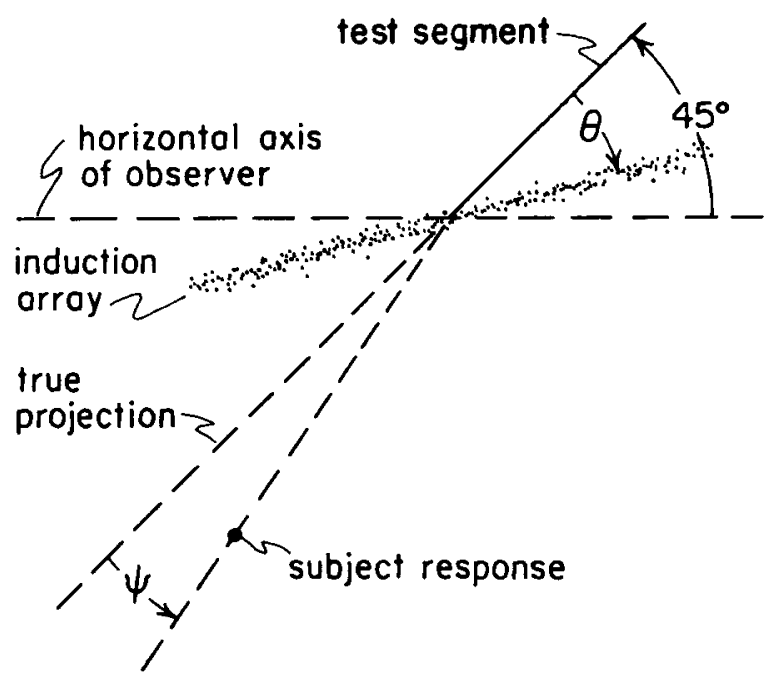

Figure 1. The visual stimulus contained either a test segment alone, or a test segment in combination with an induction array, as is shown. Subjects placed a point across the open space of the page at a point that appeared to be collinear to the test segment. The orientation of the induction array, relative to the test segment (known as $\theta$ ), produces a collinearity error $(\Psi)$, which is measured as an angle from the true projection, as is illustrated.

Procedure. On each of the stimulus pages, a test segment $\left(7.5^{\circ}\right.$ of arc long and $4^{\prime}$ of arc thick) was placed at a $45^{\circ}$ angle relative to the horizontal axis of the page. The test segment was positioned in one quadrant of the page, with the long axis of the page being oriented horizontal to the observer. The test segment was collinear to the page center, with its closest end lying at $8.3^{\circ}$ of arc from that point. Across the pages of the set, the test segment was shown in each quadrant, thus requiring the subject to make a judgment of collinearity in four directions for each stimulus configuration that was investigated.

Each induction array was positioned so that its longitudinal axis intersected the tip of the test segment. The span of the induction array was $12^{\circ}$ of arc, and the array was centered on the tip of the test segment along its length. A major variable in this experiment was the angle of this axis $(\theta)$, which was positioned relative to the orientation of the test segment. Six values of $\theta$ were used, in $15^{\circ}$

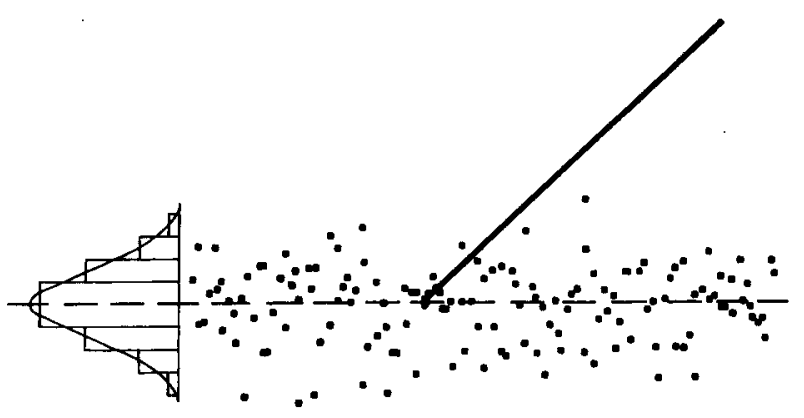

Figure 2. The induction arrays were created by scattering points at right angles to the central axis. The points were plotted on a grid with units measuring $4^{\prime}$ of arc and with one axis of the grid aligned with $\theta$. Along this axis the array contained one point at each interval of the grid, and each point was plotted at a discrete interval along the other axis by using a Gaussian probability distribution. The bin width for this distribution was much closer than is shown in the illustration, and thus the modeling that follows treats the scatter as a smooth Gaussian. 

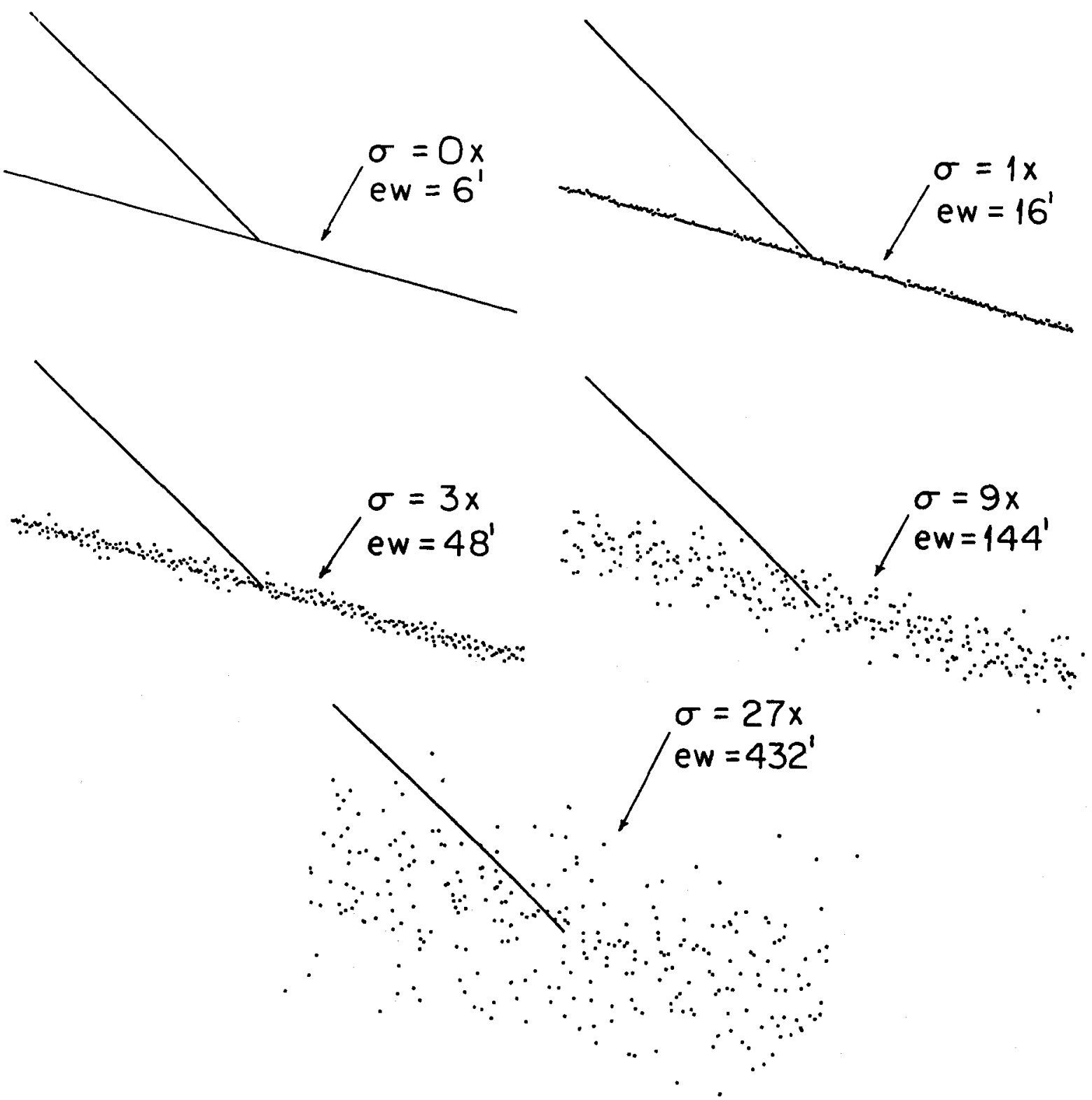

Figure 3. Five levels of scatter were used. The amount of point spread is specified by the standard deviation of the Gaussian multiplied by the dimensions of the plotting grid, where $x=4^{\prime}$ of arc. Additionally, we give the width of the band, which contains $95 \%$ of the points, and designate this as the "effective width" (ew) of the array. The dimensions of effective width are expressed in minutes of arc.

steps, and they were suitably mirrored in each of the quadrants, as described below.

The points of each induction array were approximately square, with a cross-section of $4^{\prime}$ of arc. They were plotted on an (unseen) Cartesian coordinate grid that was aligned at the specified angle; the units of the grid were $4^{\prime}$ of arc. Each grid line orthogonal to the axis contained a point; its location was assigned as a discrete probability function relative to the central axis, as is shown in Figure 2 . The spread of points in the array was determined as a random sampling from normal probability distributions, which differed in scale as multiples of the discretizing criteria $x$, which was set at 9999 of the grid scale integers. Five stimulus arrays were constructed, in which $\sigma=0 x, 1 x, 3 x, 9 x$, or $27 x$. Thus, in the stimulus configuration in which $\sigma=0 x$, all points were placed on the central axis to produce a smooth line. For $\sigma=1 x$, one third of the points were positioned away from the central axis by one grid unit or more. For $\sigma=3 x$, one third were positioned away by three units or more, and so on. The appearance of the scatter for each condition is illustrated in Figure 3.

One can also summarize the degree of scatter by giving the width of the zone that contains $95 \%$ of the points, which is designated as the "effective width" of the stimulus array. The present arrays had effective widths of $16^{\prime}, 48^{\prime}, 144^{\prime}$, and $432^{\prime}$ of arc. The fine-line stimulus had a cross-section that was $4^{\prime}$ of arc; however, by considering the effects of optical blurring, one can treat this stimulus as a Gaussian with an effective width of approximately $6^{\prime}$ of arc, as we will discuss below.

The quadrants were numbered according to standard mathematical convention, beginning in the upper right corner and proceeding counterclockwise. The angular positions of each quadrant 
were defined in a way that reflects the symmetries of human vision. Specifically, $\theta$ (from $0^{\circ}$ to $90^{\circ}$ ) was defined by counterclockwise rotation of the induction array for the first and third quadrants, and by clockwise rotation for the second and fourth quadrants. With this convention, a $45^{\circ}$ array lies horizontally relative to the observer, irrespective of the quadrant in which it is presented. At the $0^{\circ}$ orientation, the array was deleted in order to avoid occlusion of the test segment whose collinearity was to be judged. The $0^{\circ}$ condition, therefore, reflects collinearity judgments that are not biased by the presence of an induction array.

Subjects. Eight undergraduates served as the subjects for Experiment 1 . The subjects were naive with respect to the phenomenon under investigation as well as to the specific hypotheses. Each subject had normal (20/20) acuity with each eye, although some were corrected to normal with contact lenses. Each subject judged the stimulus pages in a random order with the aid of a viewing stand, following the methods of Weintraub and Brown (1986). The stand provided a viewing/marking surface, which was inclined at $15^{\circ}$ relative to the observer, and had edge stops to aid in the positioning of stimulus pages. The stand also provided a mask, with eye slots placed at a distance of $46 \mathrm{~cm}$ above the viewing/ marking surface. The horizontal alignment of the eye slots discouraged rotation of the head and thus maintained consistent orientation and distance of the stimulus material.

The subjects were told that they would be tested on their ability to judge the alignment of stimulus segments. They were instructed to "mentally extend the test segment across the page, and to mark a point which is aligned with it." They were told to select a point on the "opposite side of the page," and a zone approximately $20^{\circ}$ of arc from the tip of the test segment was indicated. Greene (1993b) has found that strength of induction remains the same irrespective of the distance that is chosen, so no additional effort was made to constrain the response. Once the subjects understood the requirements of the task, they were allowed to proceed through the stimulus set at their own pace. Most completed the set in less than $1 \mathrm{~h}$.

\section{Results}

As is indicated in Figure 1, the collinearity error $(\Psi)$ was measured as the angular departure from the true projection of the test segment. ${ }^{1}$ Previous results from this laboratory have not shown major differences in error level as a function of the quadrant in which the stimulus was displayed. Therefore, in the present analysis, for each subject the data were collapsed across quadrants, providing a mean for each combination of the two experimental factors.

Factor A consisted of the five levels of scatter, and Factor B was composed of the six orientations of the induction array. The average score for the $0^{\circ}$ position (where the test segment was presented alone) is graphically displayed, but it was not a part of the statistical evaluation of experimental effects.

The data for the 8 subjects were analyzed with a twoway within-subjects analysis of variance (ANOVA). In order to minimize the risk of Type I error, and to avoid undue emphasis of weak effects, $\alpha$ was set at .01 . The $F$ values for $\mathrm{A}, \mathrm{B}$, and $\mathrm{AB}$ were as follows: $F(4,28)=35.5$, $F(5,35)=37.0$, and $F(20,140)=7.7$. Each was significant at $p<.001$.

The means and standard errors of the mean for the 8 subjects at each of the induction angles and for the five levels of scatter are plotted in Figure 4. The angular in-

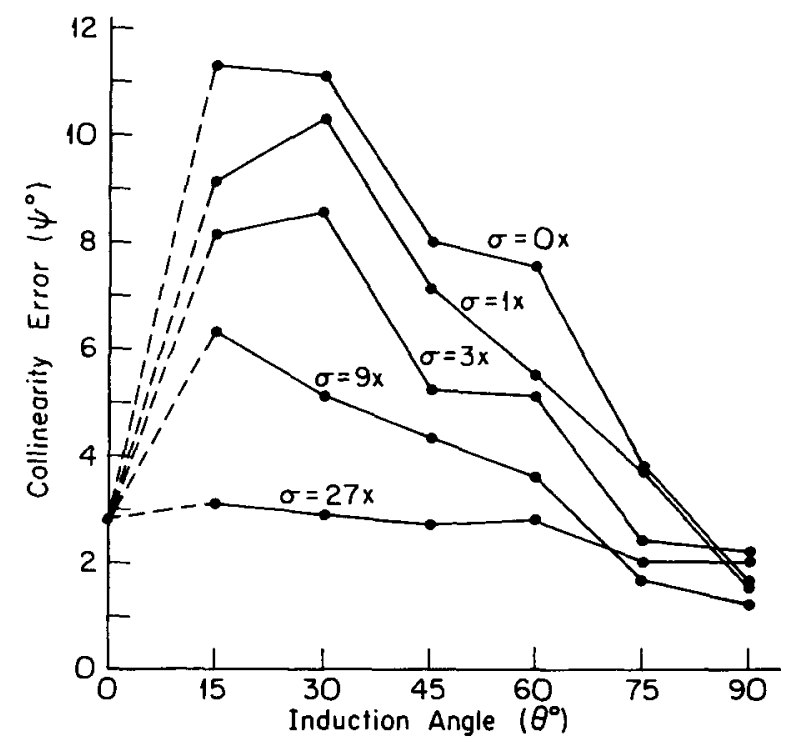

Figure 4. Each array produced angular induction effects that were a function of $\theta$ (error bars are given in Figure 5). A fine line produced the strongest angular induction, and the strength of the effect grew weaker with increasing scatter of the points.

duction process-that is, misprojection as a function of orientation of the array, is best described by considering the plot from right to left. The strength of the effect was minimal when the induction array was orthogonal to the test segment and increased as the angle became less orthogonal. Collinearity judgment in the absence of angular induction is shown by the point at $0^{\circ}$, where the test segment was presented in isolation.

\section{Modeling the Psychophysical Effects}

The amount of point scatter had a systematic effect on the induction process, in that the slope of the curve dropped progressively as the spread of the points became larger. Previous work (Greene, 1993a, 1993b, 1994) suggests that angular induction effects should be zero where the array is orthogonal to the test segment and should vary in strength as a function of the angle, according to the following formula:

$$
\Psi^{\circ}=p\left(1-\frac{\theta^{\circ}}{90^{\circ}}\right) .
$$

The model indicates that the strength of induction is determined by the size of the relative angle between the two lines $(\theta)$ and with respect to a single constant $-p$. The value of $p$ is provided at the intercept of the theoretical curve at $0^{\circ}$, essentially ignoring the collapse or indeterminate nature of the true effects at very close angles.

It is useful to respecify the equation, considering $p$ to be a normal random variable, as is shown in Equation 2. If we solve for $p$, then average across subjects and angles, we can use the observed data to estimate the size of $p$ for each of the arrays (as is shown in Equations 3 and 4). 


$$
\begin{gathered}
\Psi_{i j k}^{\mathbf{o}}=p_{i j k}\left(1-\frac{\theta_{j}^{\mathrm{o}}}{90^{\circ}}\right) \\
p_{i j k}=\frac{\Psi_{i j k}^{\mathrm{o}}}{\left(1-\frac{\theta_{j}^{\mathrm{o}}}{90^{\circ}}\right)} \\
p . k=\frac{\sum_{1}^{n} \sum_{1}^{m} p_{i j k}}{m n},
\end{gathered}
$$

where $i=1$ to $n$, the number of subjects, $j=1$ to $m$, the number of angles, $k=1$ to $s$, the number of scatter levels. Thus we average over each level of $i$ and $j$, and are provided with an estimate of the strength of $p$ for each level of $k$.

Thus, the influence of point scatter was modeled by using the value of each group mean at each angle, subtracting residual effects, ${ }^{2}$ which are reflected in the $90^{\circ}$ value, and ignoring the judgments at $0^{\circ}$. Using the estimate of $p$ for each level of point scatter, one can predict the size of the error across all levels of $\theta$ - the expected values are straight line functions. The data have been replotted against these theoretical predictions in the panels of Figure 5. The effects appear to follow the angular rule specified by Equation 1; the spread of points causes a decrease in the strength of induction at any given angle and produces a lower slope across angles.

It is noteworthy that Figure 5 suggests consistency of induction effects across all levels of point scatter. It seems clear from Figure 5 that the theoretical predictions match the observed data for arrays that have a wide scatter as well as for those having little or no scatter. This is somewhat counterintuitive, because one might expect that the rather amorphous quality of the widest band would result in a high variability of perceptual effects. Instead, it appears that the widest array produced a small but consistent amount of induction bias which was a function of $\theta$, and there was no indication that an increase in point scatter was accompanied by a decrease in the reliability of the effect.

It should be clear that $p$ is the only free parameter in the model of the angular induction effect; the value of the element depends upon the scatter of points within the stimulus array. It can be said, therefore, that $p$ provides a summary of the induction potential for any given stimulus, and for convenience we describe it as measuring the "strength" of a given stimulus configuration. For the present data, we have extracted the value of $p$ for each stimulus array, normalized each value relative to the fine-line stimulus, and have plotted the resultant values in Figure 6 as a function of point scatter.

Figure 6A shows the decline in induction strength using a linear scale of effective width, and Figure 6B uses a logarithmic scale. It may be significant that the decline of strength plots as a fairly straight line when the dimensions of the stimulus array are transformed by taking the logarithm.

\section{EXPERIMENT 2}

\section{Rationale}

The results of Experiment 1 show that the strength of angular induction is a function of the degree of point scatter. It is possible that the net density of the points is registered by the perceptual system. If so, the impact of a scattered set of points should be equivalent to a blurredline stimulus, which is a gray band in which the luminance falls off smoothly as a function of distance from its central axis. The most pertinent comparison would be between two sets-one in which the points were scattered by using Gaussian probability distributions (as in Experiment 1), and the other with corresponding Gaussian profiles of luminance gradient. Each member of the latter set is described as being a Gaussian blur segment, or, more simply, as a blurred segment.

A second issue relates to whether the strength of induction is determined intrinsically by the degree of scatter, or whether it might relate to the degree of correspondence between the induction array and the test segment. In other words, it is conceivable that the two are registered by "channels" that are specific for the dimensions of the stimulus component. Thus, the test segment would have its major influence on a narrow channel, but the induction array would be registered by a channel that is either narrow or wide, depending on the degree of scatter. One might hypothesize that there is preferential interaction among the channels that are comparable (or similar) in size. If so, the decrease in strength of induction with increasing point scatter may be due to a mismatch of channel size. For convenience, we will refer to this concept as channel coupling. To evaluate the channel-coupling hypothesis, we constructed a stimulus set in which the points of both stimulus components were dispersed; each level of scatter for one was presented against the various levels of scatter of the other. Thus we adopt the term test array to describe the stimulus which is judged, and specify that the subject's task is to evaluate alignment of the central axis of this array.

\section{Method}

Stimuli. Unless otherwise stated, stimulus and test conditions were identical to those used in Experiment 1. There was no indication that quadrant had an influence on the size of induction, so the stimulus material was restricted to Quadrant 2 (upper left corner of the page). Similarly, the results of Experiment 1 indicated that the effects follow the same angular rule for various values of $\theta$, so we positioned each of the test arrays at a $45^{\circ}$ angle.

Procedure. A 10-page set was constructed for the comparison of point scatter with the blurred segments. The pages of the pointscatter set were printed with a standard 300-dpi LaserWriter. The dimensions of the arrays were the same as those in Experiment 1; effective widths were $4^{\prime}, 16^{\prime}, 48^{\prime}, 144^{\prime}$, or $432^{\prime}$ of arc. The pages of the blurred-segment set were printed commercially on a Linotronic machine that had a nominal resolution of 2,540 dpi with 256 

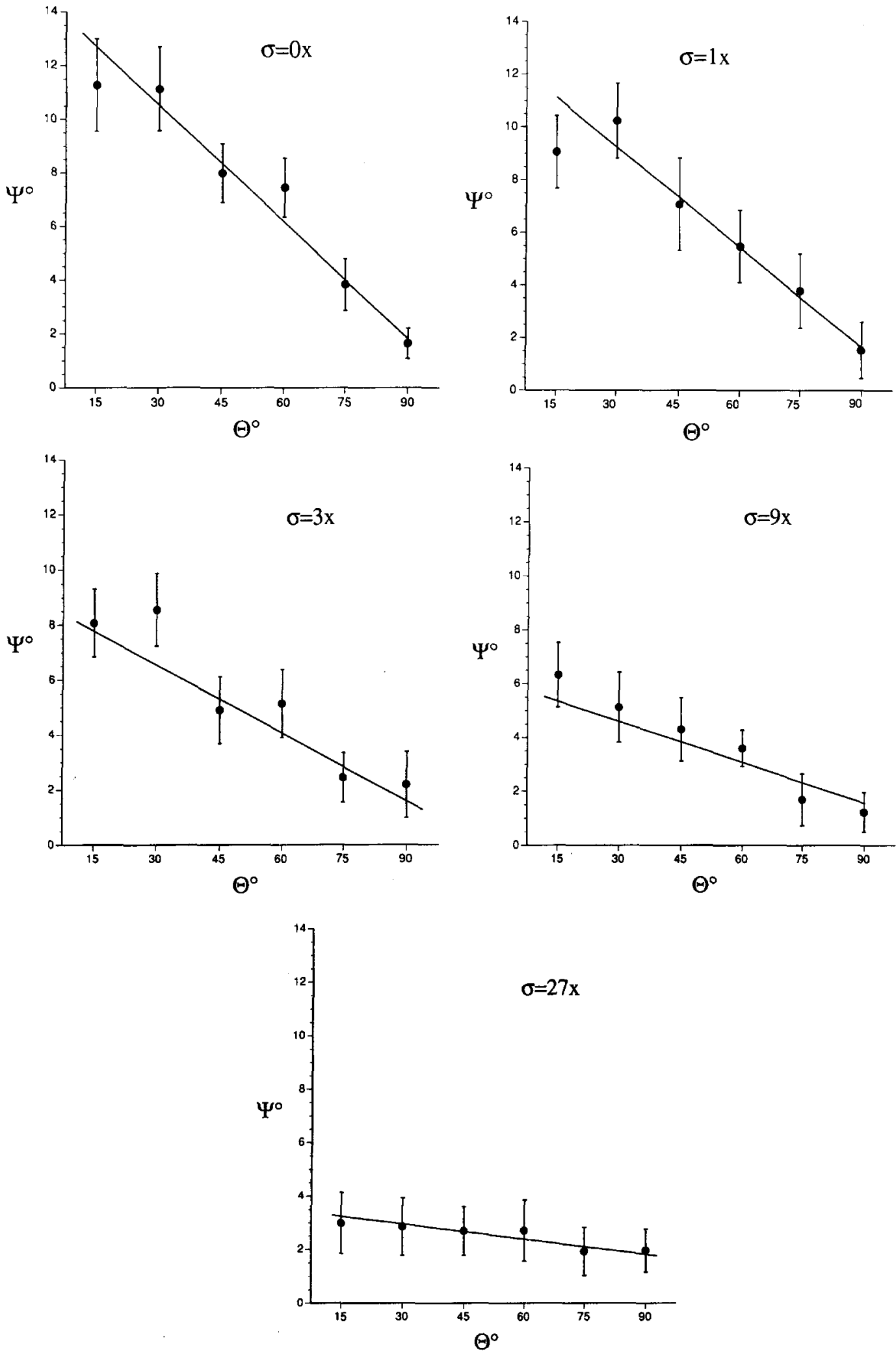

Figure 5. The means for each level of scatter are shown in separate panels, along with the standard error of each mean and the theoretical predictions, which were derived from a model of the induction effect. Increasing the scatter of points appears to change the slope of the functional relation between $\theta$ and $\Psi$. Note that the match between prediction and data is similar, irrespective of the width of the array. This suggests that the scattering of points alters the strength of induction, but not the consistency of angular effects. 

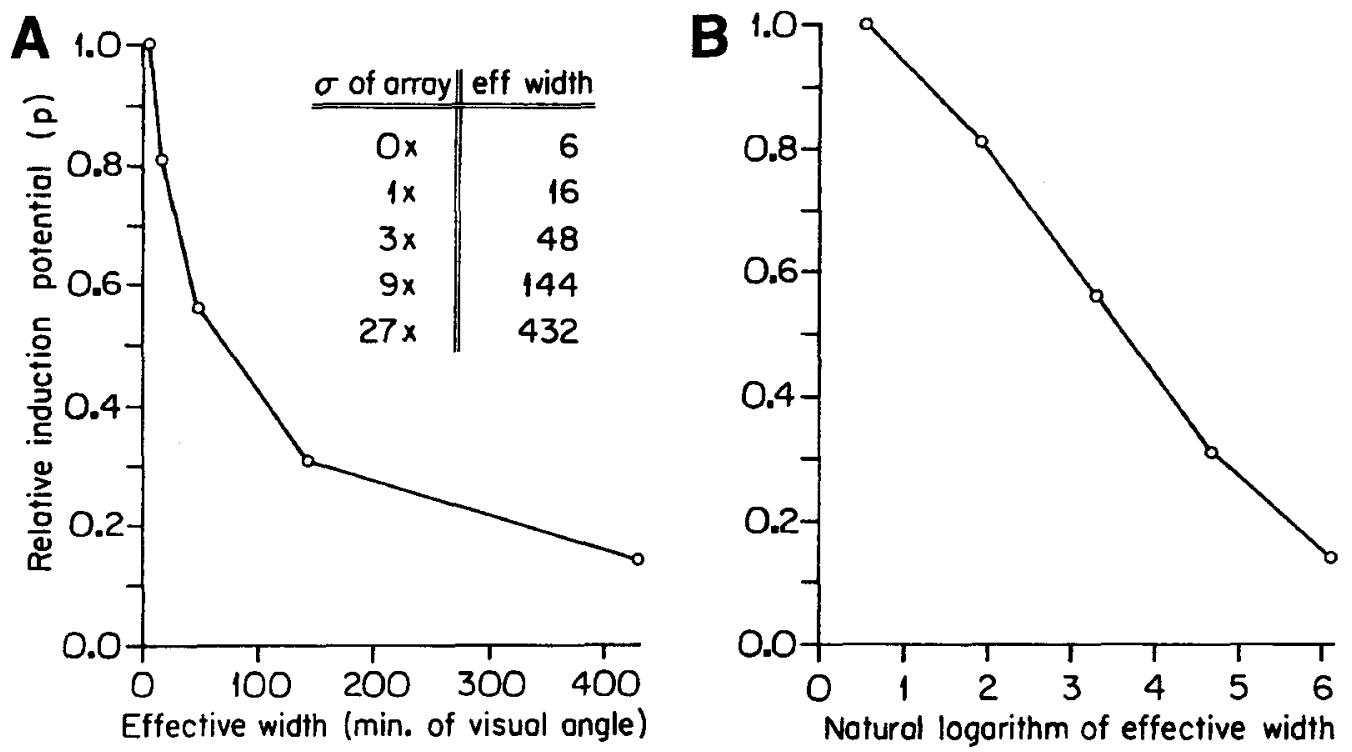

Figure 6. (A) The perceptual model contains a single free parameter (p), which provides a summary of the potential for a stimulus to produce induction effects. From the experimental results, we have extracted the relative value of $p$ for each stimulus array and have plotted this value as a function of the width of that array. (B) The relative values of $p$ are plotted with logarithmic transformation of effective width.

levels of gray. This set consisted of five pages--each had an effective width that corresponded to one of the values given above, except for the smallest value, which was set at $6^{\prime}$ of arc. The reason for this adjustment requires a bit of explanation. It should be remembered that in order to simplify the terminology, we have described the narrowest segment as having an effective width of $4^{\prime}$ of arc. Although this is indeed the dimension of the segment, the term effective width relates to the spread of a Gaussian distribution-the span that captures $95 \%$ of the area under the curve. To create the corresponding Gaussian blur segment, we must decide what dimension is likely to produce an effect that is the same as that of a uniform square wave with $100 \%$ contrast and a width of $4^{\prime}$ of arc. Various considerations-most notably the fact that optical blurring of the image spreads a sharp edge by about $1^{\prime}$ of arclead to the conclusion that the narrowest blurred segment should have an effective width of $6^{\prime}$ of arc.

In keeping with view that the receptive field might respond to the net density of the point scatter, the overall contrast of the blurred segments was specified as being equal. Thus, as the width of the Gaussian profile was increased, the height of the curve was lowered. For the present stimuli, with effective widths of $6^{\prime}, 16^{\prime}$, $48^{\prime}, 144^{\prime}$, and $432^{\prime}$ of arc, the contrast values at the center of the curves were $100 \%, 37.5 \%, 12.5 \%, 4 \%$, and $1.4 \%$, respectively.

The stimulus set for testing channel coupling presented test and induction arrays in the various combinations of width, specifically, effective widths of $4^{\prime}, 16^{\prime}, 48^{\prime}, 144^{\prime}$, and $432^{\prime}$ of arc. Thus, a given level of scatter for each test array was combined for each level of scatter for the induction array, yielding a total set consisting of 25 pages.

Subjects. Nine subjects judged each stimulus set three times. The order of material within a set was random, and the sets were rerandomized between judgments. Other test conditions were the same as those in Experiment 1.

\section{Results}

To compare the induction effects of point-scattered arrays versus blurred segments, the data for the three judgments were averaged, and these values were used for the statistical analysis of results. The data were eval- uated with a two-way within-subjects ANOVA (again, $\alpha=.01$ ). Factor A (dimension of the array/blur) was found to produce a significant effect $[F(4,32)=18.73$, $p<.001]$. Factor B (comparison of point scatter vs. blurred segments) was not significant $[F(1,8)=2.05]$, and the interaction between $\mathrm{A}$ and $\mathrm{B}$ was nonsignificant as well $[F(4,32)=1.33]$.

A supplemental analysis was done to specifically test whether the induction effect of the narrowest Gaussian (effective width of $6^{\prime}$ of arc) might be different from that produced by the fine-line segment (effective width of $4^{\prime}$ of arc). A matched-sample $t$ test proved to be nonsignificant $[t(8)=0.95]$. Thus, in the following comparison of the data from Experiment 1 to the model, we can treat the fine-line stimulus as being equivalent to a Gaussian having an effective width of $6^{\prime}$ of arc.

The means and standard errors for the two treatment conditions are shown in Figure 7. It is clear that the strength of induction decreased as a function of increasing scatter of the points and spread of the Gaussian blur. The strongest effects were produced by arrays that had the smallest effective widths, and the influence declined as the dimension of the scatter/luminance profile was increased. The small differentials in the two curves were not significant, as we have indicated.

The second issue to be resolved in Experiment 2 was whether the strength of induction might be jointly determined by the widths of the induction and test arraysthe channel-coupling hypothesis. To briefly reiterate, one might well argue that a given array will have its greatest influence on a "channel" that has a corresponding dimension, and that the strength of the interaction between channels is greatest when they are similar in size. For the statistical analysis, we designate the scatter 


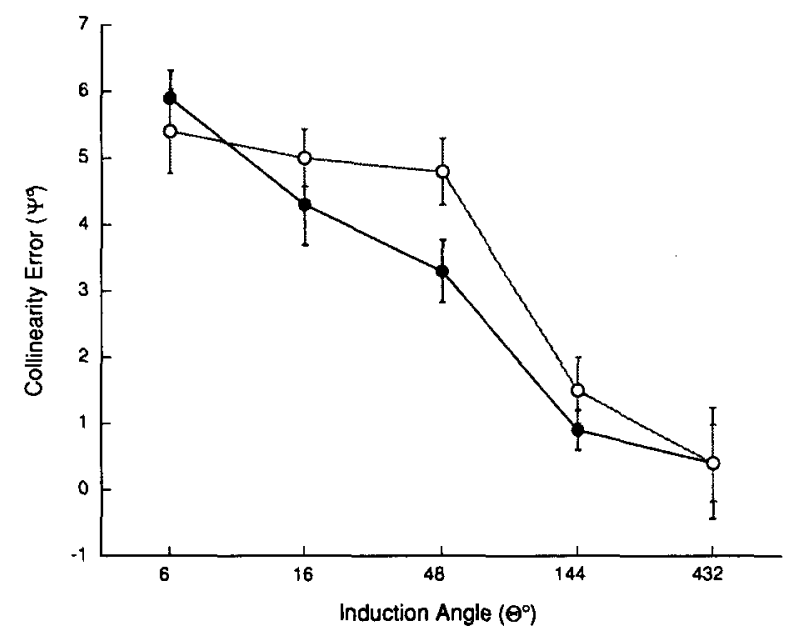

Figure 7. In the first half of Experiment 2 we compared the induction effects, which are produced by arrays of scattered points (filled circles), with the effects produced by Gaussian blurs (open circles). There was no significant difference between the two treatment conditions, and it can be seen that both decline monotonically as the dimension of the induction array is increased.

of the induction array as Factor A, and the scatter of the test array as Factor B. The prior results suggested that Factor A should be significant. We have no basis for a prediction with respect to Factor $B$, but would expect a significant interaction between $A$ and $B$ if some portion of the overall effect is due to channel coupling.

The data for the three repetitions of the experiment were combined, and the results were analyzed with a two-way within-subjects ANOVA. Factor A (induction array spread) was significant $[F(4,32)=6.64, p<.001]$; Factor $\mathrm{B}$ (test array spread) was significant $[F(4,32)=$ $5.77, p<.01]$; and the interaction between the two was also significant $[F(16,128)=3.15, p<.01]$.

As is shown in Figure 8A, scatter of the induction array produced effects that were similar to those found in Experiment 1. The strongest bias was produced by the fine-line segment; the influence declined monotonically as a function of increasing scatter. This was not the overall pattern, however, with increasing scatter of the test segment. As can be seen in Figure 8B, the relative susceptibility to induction was a curvilinear function of scatter. The arrays with widths of $16^{\prime}$ and $48^{\prime}$ of arc were more easily biased than were those that had a larger or smaller degree of scatter. This is an interesting result in itself, and one for which we have no ready explanation. If we had been forced to make a prediction in advance of the experiment, we might have hypothesized that susceptibility to bias would be a direct function of variability of judgments, with the wider arrays being less stable and thus more easily influenced by the induction process. This is not supported by the data. In general, there was no indication that the degree of variability of judgments was related to the size of the induction effect. Indeed, the only notable difference in variability was for the array that was $432^{\prime}$ of arc, and this stimulus showed no susceptibility to induction.

At a minimum, one would not be able to argue for channel coupling unless the interaction of the treatments was significant. However, the statistical power
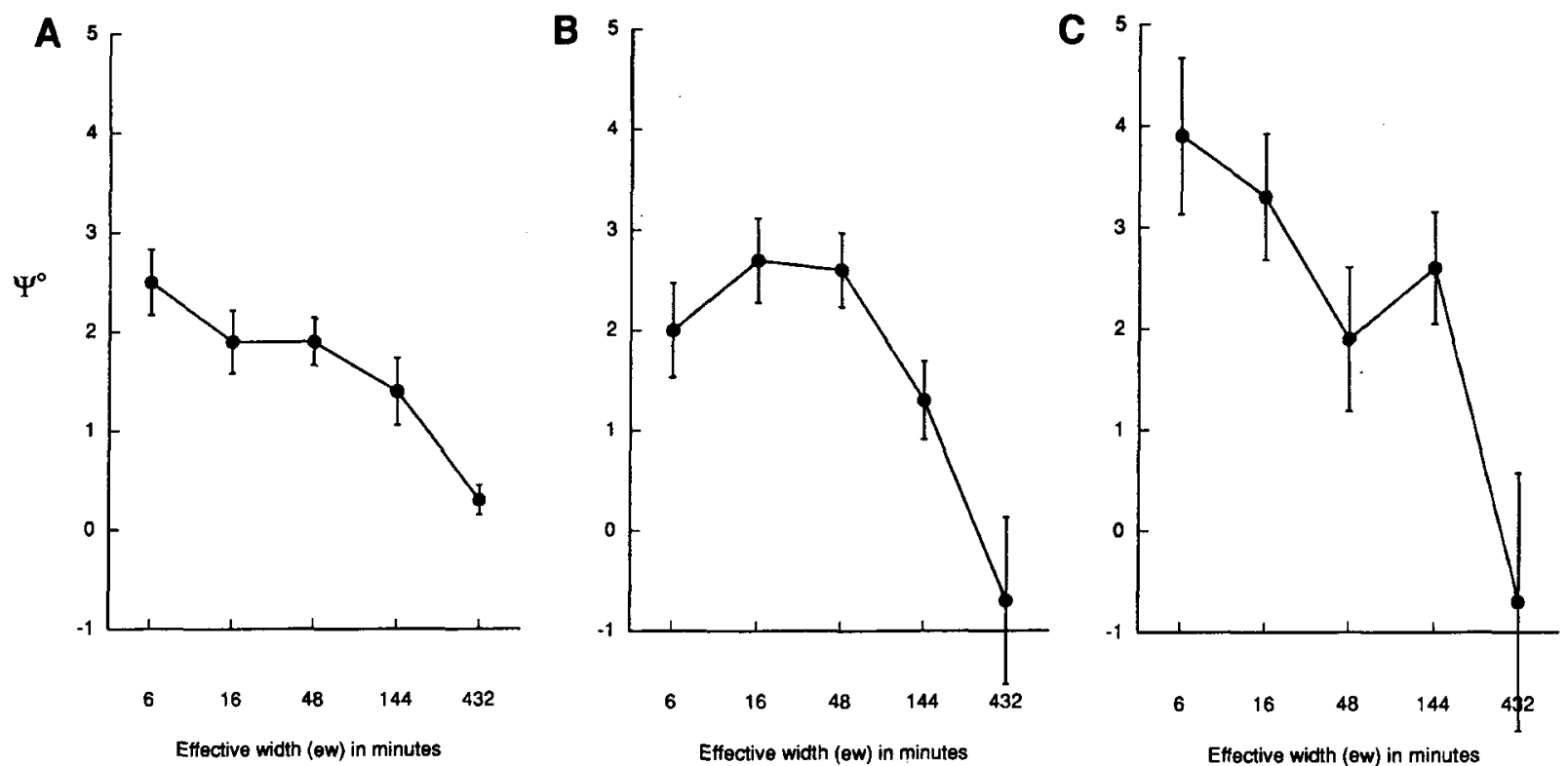

Figure 8. In the second half of Experiment 2 we varied the scatter of the test array as well as that of the induction array, forming a $5 \times 5$ matrix of treatment combinations. Panel $A$ plots the "marginal" means from differential scatter of points in the induction array, averaged across all levels of the second treatment condition. Panel B plots the marginal means for scatter of points in the test array. It is clear that an increase in scatter produces a monotonic decline in the strength of an induction array, whereas an increase in scatter of the test array has a curvilinear effect on susceptibility to induction. Panel $C$ plots the diagonal of the $5 \times 5$ matrix $\longrightarrow$ that is, where the dimensions of the test and induction arrays are the same. Although the statistical analysis provided support for a "channel-coupling" hypothesis, the sharp decline of induction strength with increasing scatter suggests that coupling is not the controlling factor. 
of the repeated measures paradigm can show significance even though the effects are relatively weak. This appears to be the case here; the formal analysis reported previously does indicate a significant interaction between the two factors, but the pattern of data does not suggest a major role for channel coupling. This point is best illustrated by plotting the diagonal of the treatment matrix - that is, where the scatter of the induction array was the same as that for the test array. Figure $8 \mathrm{C}$ shows the degree of induction that resulted from this matching. If channel coupling provided major control over the strength of induction, one should expect the induction to be approximately the same, irrespective of scatter, or at least to show a decline that was less than the effect generated by unmatched conditions. As can be seen in the plot of Figure $8 \mathrm{C}$, there is substantial decline in induction effect with increasing scatter. Thus, the strength of an induction array is a function of its scatter whether it is paired with a narrow test segment, as in Experiment 1, or with a test array that has a corresponding degree of scatter. We submit, therefore, that although one can insist that channel coupling has a role in this process, it does not appear to be a dominant factor.

\section{MODELING BIOLOGICAL SUBSTRATES}

We assume that perceptual states are determined by interactions among neurons, but the way that the brain mediates judgment and bias of orientation is far from clear. Nonetheless, it may be useful to formulate a specific model for determining how the induction array is encoded and whether the response of an encoding system corresponds to the strength of perceptual influence, as it is reflected in the value of $p$ in Experiment 1 .

For this purpose, we start with the assumption that the source of geometric illusions related to angular induction is located as early as the primary visual cortex; therefore, the results of systematic variation of stimuli are dominated by changes in the activity of these neuron populations. We assume that the orientation of a contour line controls the activity of individual neurons, and we adopt (with modification) the concept that orientation selectivity is determined by the sensitivities of the classic "receptive field," in which an elongated excitatory zone is flanked by inhibitory zones (Hubel \& Wiesel, 1962). The modification is provided by the results of Jones and Palmer (1987a, 1987b), which indicate that the strength (and direction) of influence across these zones can be modeled as a Gabor function. Thus, in the present analysis we will concentrate on the response properties that should derive from a receptive field with such a design.

The results of Jones and Palmer (1987a, 1987b) indicate that many neurons have an excitatory zone that is bordered by either one or two inhibitory flanks. It is presumed that the field with a single flank would be most effective at perceiving an edge, and the one with two flanks would respond best to a line segment. We restrict

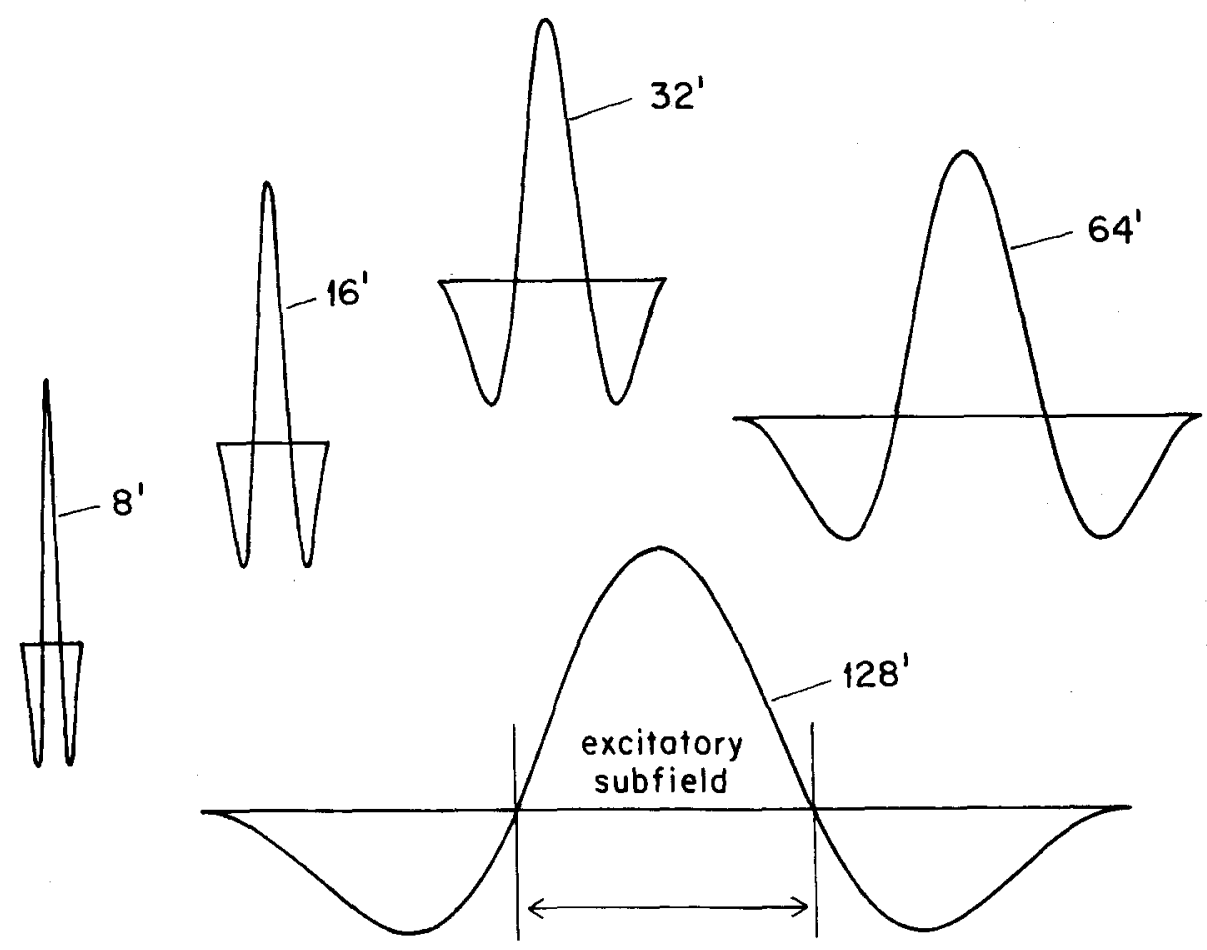

Figure 9. Neuron responses were modeled by assuming that the influence along the long axis of the array is constant, and that responsiveness across the width of the receptive field can be summarized by a one-dimensional Gabor function. The members of the set are identified by the width of the excitatory subfield; this field is labeled, and its span is indicated on the largest Gabor. The entire Gabor function contains the two inhibitory flanks, and thus it is three times the width of the excitatory subfield. 
our attention to the the latter family, known formally as even symmetric Gabor wavelets.

As simplifying assumptions, the induction array can be considered to be redundant along its long axis, which allows the stimulus to be represented by the Gaussian function that specifies the scattering of points. ${ }^{3}$ Similarly, the receptive field is modeled by using a onedimensional Gabor, which specifies the excitability profile across the short axis. ${ }^{4}$ Thus, the Gabor expresses the degree to which a line would excite the neuron as a function of displacement across the short axis and, more relevant to the present analysis, its response to the dispersal of points as reflected in the stimulus Gaussian.

A more comprehensive list of our receptive field modeling assumptions follows:

1. The Gabor function is defined as the product of a cosine wave and a Gaussian distribution (not to be confused with the stimulus Gaussian).

2. We assume wavelets (Mallat, 1989), in which the field is delimited to $3 / 2$ of the cosine, a central excitatory half-wave flanked by two inhibitory half-waves.

3. The Gaussian component is centered on the cosine component, thus creating an even symmetrical field.

4. The width of the components are adjusted so that two standard deviations on each side of the Gaussian correspond to the endpoints of the cosine component.

5 . The response of the receptive field is evaluated with the stimulus centered upon the field.

6 . The response at each point in the receptive field is modeled as the product of the Gabor and the stimulus intensity at each point.

7. The receptive field responds as a linear system, in which the overall response to a stimulus is the simple sum of the product described in (6) at each location, and at each location this response is dominated by the response of the cell with optimal orientation with respect to the stimulus orientation. ${ }^{5}$

We have chosen receptive field sizes with an eye to biological plausibility. R. L. DeValois, Albrecht, and Thorell (1982) report that the optimal spatial frequency response of simple cells has a range of about five octaves, with the mean at or slightly above 2 cycles/degree of arc ( $30^{\prime}$ of arc for the excitatory portion of the field). For convenience, we have modeled the data by using a set of five Gabor functions with excitatory subfields of $8^{\prime}, 16^{\prime}, 32^{\prime}, 64^{\prime}$, and $128^{\prime}$ of arc (illustrated in Figure 9).

Response curves were generated by multiplying stimulus Gaussians, which ranged in effective width between $6^{\prime}$ and $432^{\prime}$ of arc, against each member of the set of Gabor functions. The curves were normalized (setting the largest value among all the fields to 1 ), and the resulting values are plotted as solid lines in Figures $10 \mathrm{~A}$ and $10 \mathrm{~B}$, using linear and logarithmic scales on the abscissa. Each of the five solid lines provides the relative response for a given size of Gabor as a function of the width of the stimulus Gaussian.

It may be easiest to describe the influence of changing stimulus and receptive field parameters with reference to Figure 10A. The tallest line predicts the response of the model where the Gabor has an excitatory field of $8^{\prime}$ of arc, beginning with a stimulus Gaussian with an effective width of $6^{\prime}$ of arc and ending with one that spans $432^{\prime}$ of arc. It should be remembered that the initial point of this curve has been set to 1 ; all other interactions are proportional to that value, and they will be referred to as a percentage of the maximal response.

It is clear that the impact of the stimulus Gaussian drops sharply as effective width is increased, becoming
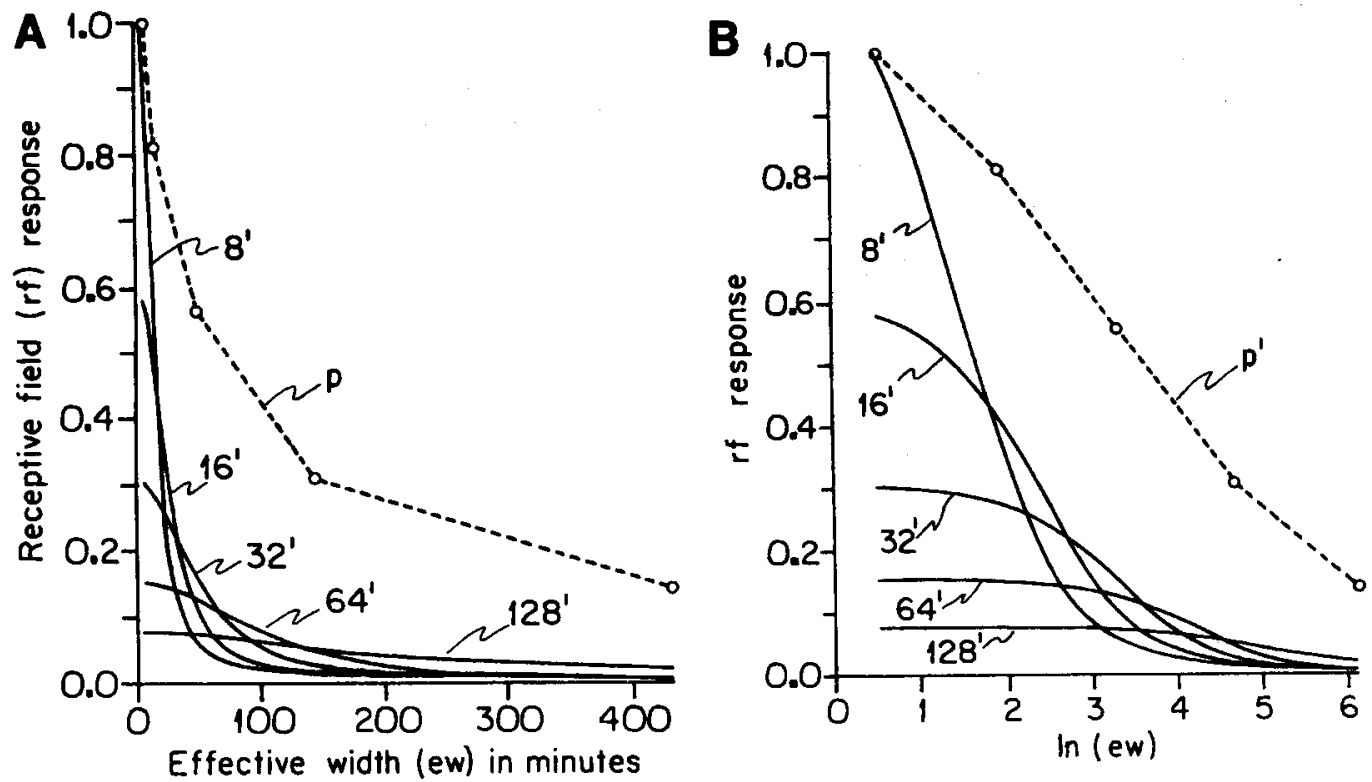

Figure 10. (A) The stimulus arrays were modeled as Gaussians, which are equal in area. Relative responses from each Gabor function were calculated for the range of stimulus Gaussians between $6^{\prime}$ and $432^{\prime}$ of arc; these are shown as solid lines. The $p$ curve from Figure $6 \mathrm{~A}$ is provided to facilitate comparison. (B) Relative responses of the Gabors are plotted with logarithmic transformation of effective width. The transformed $p$ curve from Figure $6 B$ is identified as $p^{\prime}$. 
only $4 \%$ where the width is $20^{\prime}$ of arc. The other curvesthat is, where the excitatory fields of the Gabors are $16^{\prime}$, $32^{\prime}, 64^{\prime}$, and $128^{\prime}$ of arc-show similar patterns of decline with increasing width of the stimulus Gaussian. As anticipated, the narrow stimulus has considerably less influence upon the wider receptive fields; for example, the response of the $128^{\prime}$-of-arc Gabor to the 6'-of-arc stimulus is only $8 \%$. Perhaps less expected are the low response levels (10\% or less) from all receptive fields where the stimulus has an effective width of $20^{\prime}$ of arc or more. The lack of impact of these stimuli is undoubtedly due to the low contrast of any array for which the points have been scattered into a great deal of open space. In other words, in keeping the number of points constant and increasing the spread among the points, we drop the contrast of the resulting stimulus. ${ }^{6} \mathrm{~A}$ wider receptive field may be provided with a set of points that fall completely within its excitatory zone, yet it may not give much response because of the intrinsically low contrast level of the stimulus material.

The relationship between the biological model and the psychological results is far from obvious, and the issues need to be framed. On the psychological side, the index of induction strength $(p)$ is determined by the relative collinearity of the points. The normalized strength of $p$ is $100 \%$ when the points form a line that has no scatter, and the strength decreases systematically as a function of spread away from the central axis. To facilitate comparison with the biological model, the linear and logarithmic plots of the $p$ curve are shown in Figures 10A and 10B.

One can ask whether the decline in strength of the $p$ curve corresponds to the decreased impact of the array upon a receptive field with a fixed dimension. In other words, one can consider the hypothesis that each of the stimulus arrays is detected by one size of receptive field, and the decline in the $p$ curve is determined by the level of response from that field. To evaluate this possibility, one thus examines whether any of the solid lines in Figures $10 \mathrm{~A}$ or $10 \mathrm{~B}$ have a shape that is similar to the corresponding $p$ curve. Although each receptive field response shows a decrease with increasing width of the stimulus Gaussian, none provide a close match. Further, it seems unlikely that using a smaller or larger receptive field would improve upon this outcome, because the mismatch (except where the normalization has forced both values to unity) relates to the overall amplitude of response to the narrow stimulus Gaussian as well as to the rate of decline as the stimulus is made wider.

An alternative view of receptive field activity suggests that the fields "self-select" through mutual inhibition, so that the one that is most sensitive to a particular stimulus becomes dominant. If this is the case, one could argue that the psychological response of a given stimulus is determined by the most active field. In the context of the present model, for each stimulus width along the abscissa, the field showing the greatest response would be considered to be dominant. Thus, the overall response from the fields would be reflected by the upper envelope of the curves shown in Figures $10 \mathrm{~A}$ and 10B. For linear and logarithmic values alike, it is clear that a curve that follows this envelope provides a match to the $p$ curve that is only marginally better than that provided by the responses from each individual field.

Therefore, within the limits provided by our initial assumptions, the psychological influence of the arrays is not directly modeled by the selective activation of a particular receptive field, nor by a "winner-take-all" tradeoff among receptive fields. The next question becomes whether a modest change in the initial assumptions would
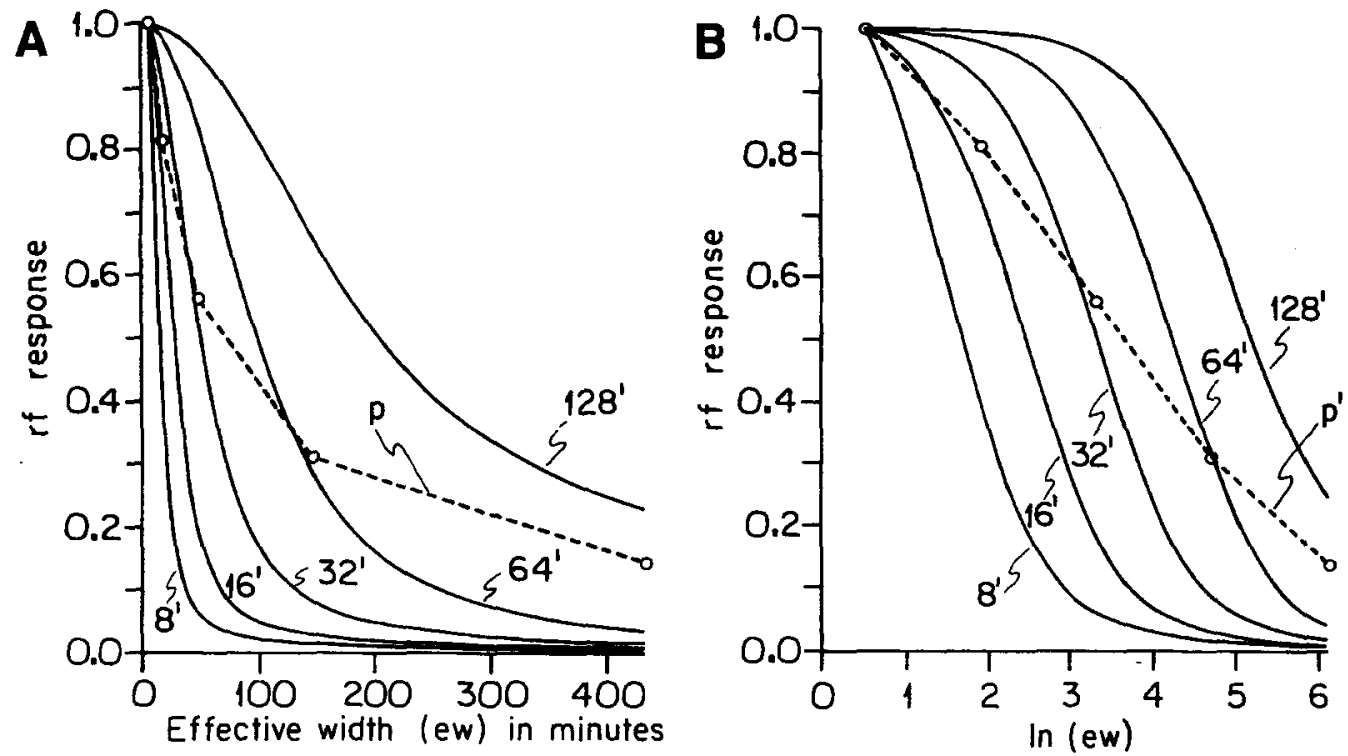

Figure 11. (A) The response of each Gabor function has been normalized to 1, which corresponds to the assumption that the fine-line stimulus produces the maximal response, irrespective of the size of the receptive field (rf). (B) A logarithmic transform of width results in a family of sigmoid curves that yields a linear plot through a sizable range of point scatter. 
result in a better match between receptive field responses and the $p$ curve that reflects the induction strength of a given stimulus.

The greatest benefit may be derived from increasing the general response levels of the receptive fields, thus compensating for the low contrast that characterizes the stimulus array. One might defend the view that neurons are capable of dynamic adjustment of their range of response, as discussed by Carandini and Heeger (1994), and/or that subpopulations exist, which are dedicated to the analysis of low-contrast material. To implement this assumption, we have normalized each curve individually so that the fine-line stimulus is seen as providing maximal activation of the receptive field irrespective of its size. The adjusted curves are shown in Figures $11 \mathrm{~A}$ and $11 \mathrm{~B}$ for linear and logarithmic scales, respectively.

None of the curves of Figure 11 provide a close match to the decline in strength of $p$ as point scatter is increased. It may be noted, however, that the general behavior of the receptive fields, as is shown in the logarithmic plot of Figure 11B, bears some similarity to the linear effects shown by the $p$ curve. The decline in response of the $8^{\prime}$-of-arc receptive field, for example, is approximately linear for stimulus widths between $6^{\prime}$ and $12^{\prime}$ of arc, and the largest receptive field $\left(128^{\prime}\right.$ of arc) gives a roughly linear response between $42^{\prime}$ and $432^{\prime}$ of arc. This invites speculation that the overall psychological response is determined by a tradeoff of relative control within the low-contrast fields, or may be generated as a combined, so-called "ensemble" response.

If perception is mediated by the activity of an ensemble, one is confronted with a number of possibilities for how the members of the ensemble might combine their effects. Averages come in many forms, and if one allows for weighted averages, the number of possible outcomes is considerable. The most direct concept is that responses of the members of the ensemble are summarized by a simple mean. Figure 12A plots the mean of the five curves at each dimension of stimulus width, and Figure $12 \mathrm{~B}$ shows this mean in relation to the $p$ curve. A similar correspondence would be shown if the means of the untransformed values were plotted against the $p$ curve shown in Figure $11 \mathrm{~A}$. We find the degree of match to be surprising, especially given the fact that we made no effort to select receptive field size or to adjust scaling in order to optimize the fit.

Perhaps the least obvious result of the modeling is the demonstration that the complex curves that are derived by multiplying a Gaussian with a Gabor form a sigmoid family when the dimensions of the Gaussian are expressed in logarithmic units and the response is adjusted for low contrast. Further, the average of this family yields a fairly linear plot across more than six octaves of width of the stimulus Gaussian. ${ }^{7}$

\section{DISCUSSION}

The goal of the present study was to evaluate the substrates of angular induction with respect to the composition of a nonclassical stimulus that produces a classical perceptual error. In most of the prior studies, a line segment has been used as the induction stimulus, and its orientation and/or position has been varied in relation to the test segment whose alignment or orientation is being judged (Andrews, 1967; Bouma \& Andriessen, 1970; Carpenter \& Blakemore, 1973; Greene, 1993a, 1993b, 1994; Greene \& Pavlov, 1989; Weintraub \& Krantz, 1971; Wenderoth, Parkinson, \& White, 1979). Using such
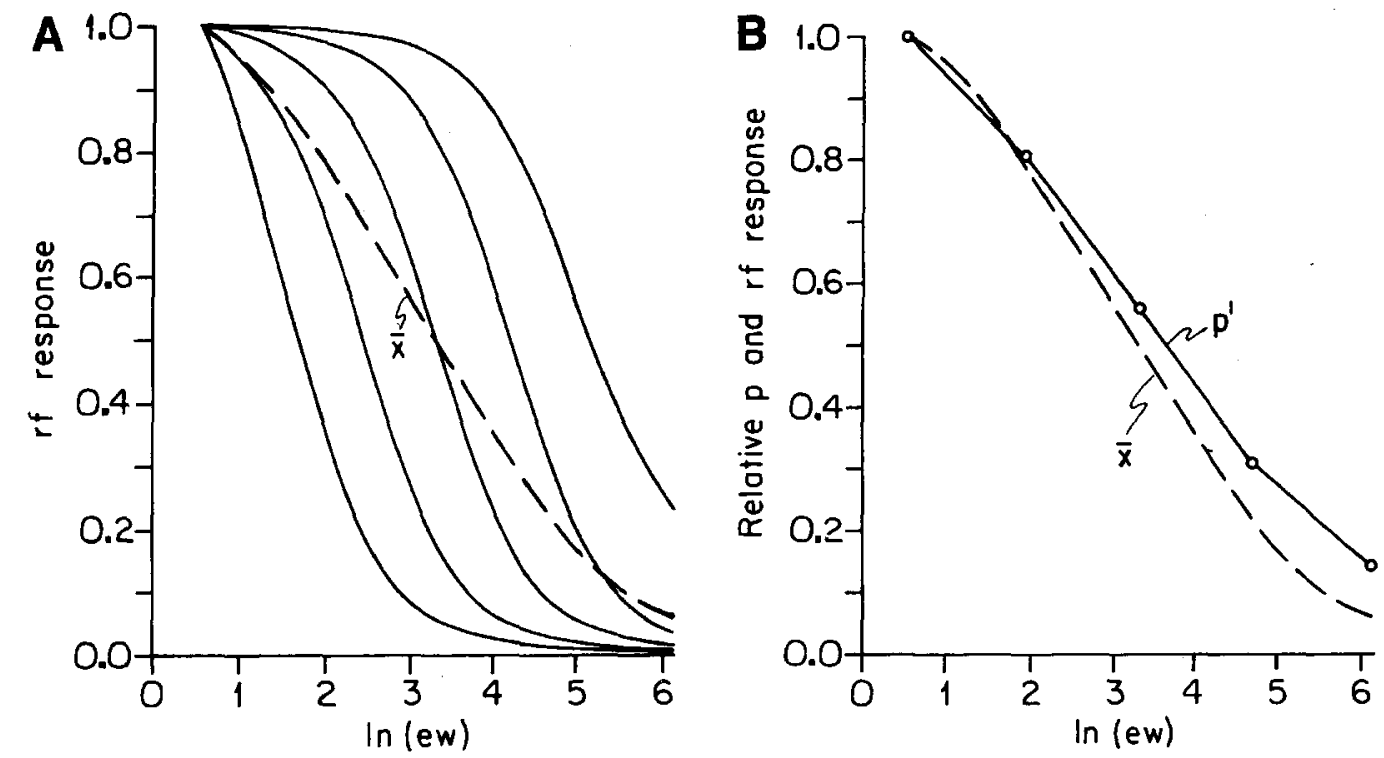

Figure 12. (A) It is possible that a set of receptive fields ( $r$ ) encodes visual stimuli as an ensemble. The dashed line shows the mean response of the Gabor functions. (B) The mean response of the Gabors is fairty similar to the $p$ curve, which describes the decrease in induction strength as the scatter of points is increased. It is conceivable that the mean response of an ensemble determines the strength of the induction effect that it can exert. (ew, effective width.) 
lines, it has been found that the amount of perceptual bias is a function of the relative angle $(\theta)$ between the test and induction segments.

The present results support the conclusion that the size of the collinearity error $(\Psi)$ is dependent upon the value of $\theta$. In addition, we have found that the functional relations are preserved when the points that comprise the induction line are scattered at right angles to its axis, and that the strength of effect decreases as a function of the amount of displacement. With respect to angular induction, therefore, the array of scattered points appears to operate as a unit-as though the points were activating a receptive field whose axis was centered across the tip of the test segment. The relative angle between the test segment and this axis thus becomes the definition of $\theta$, and $\Psi$ continues to reflect the influence that is found when a line segment is used to produce the induction. As reported previously (Greene, 1993a, 1993b, 1994), the strength of the effect can be summarized by a single parameter, $p$, which is part of the equation that relates $\theta$ to $\Psi$. The present results indicate that $p$ decreases as a function of scatter; the decline is orderly and plots as a straight line when the spread of the points is scaled by using a logarithmic transform.

The effect of scattering the line points can be seen in Figure 5; the differential of induction strength is shown by the change of slope in the predicted line. It is noteworthy that the scatter of data points about the predicted line is similar, irrespective of the dimensions of the array. This encourages the view that the individual points are registered by a single processing system that determines the judgment bias. In other words, the arrays of points have an impact that is very much like the effect of a line stimulus, and the consistency of their effect is not reduced by an increase in scatter. It may be reasonable, therefore, to suggest that they are encoded by a general contour-analysis system, in which scattered points are perceived as a "weak contour" with respect to angular induction. This possibility receives some support from Experiment 2, where it was found that the induction from Gaussian blurs is comparable to the effect of point scatter.

A case can be made that the psychological data should bear a close relationship to receptive field response. The task demands were equivalent across all pages of the stimulus set, and most aspects of the stimulus itself, such as the length of the array, were the same. The single stimulus characteristic that controlled and determined the strength of induction was the degree of scatter of the points within the array. Almost as a matter of definition, any influence of this manipulation must reflect the process by which the contour is encoded. If this point is granted, the pertinent issue is how to properly model the response of the receptive field(s).

A number of researchers have suggested that receptive fields of many neurons can be modeled as Gabor functions (Daugman, 1985; Field, 1987; Field \& Tolhurst, 1986; Jones \& Palmer, 1987a; Kulikowski \& Bishop, 1981a; Kulikowski, Marcelja, \& Bishop, 1982;
Marcelja, 1980; Sakitt \& Barlow, 1982; Watson, 1983). These functions conform reasonably well to much of the data, using stimuli as diverse as drifting sinusoidal gratings, composite gratings, fine lines that are flashed or moved, and even a minimal cluster of points that elicit responses as a function of their relative alignment. Additionally, for many of the cells that have been studied, the overall activity is predicted by assuming linear summation of response from the subfields (Andrews \& Pollen, 1979; Jones \& Palmer, 1987a; Kulikowski \& Bishop, 1981 b; Movshon, Thompson, \& Tolhurst, 1978; Tolhurst \& Dean, 1987).

The results of our biological modeling suggest that the strength of the psychological effects, as reflected by the change in $p$ as a function of stimulus dimension, is not predicted by basic luminosity response curves. The calculated output for a small receptive field was high for the narrow stimulus array (the $6^{\prime}$-of-arc Gaussian, which was used to model the line), but its activity fell off quickly as the points were scattered away from their central axis. Wider receptive fields gave predictably lower response profiles. None of these predicted response curves matched the contour of the $p$ curve; this result was most obvious in the $\log /$ linear plot of the data. It seems unlikely, therefore, that the system uses a single size of receptive field to respond to the stimulus array and to produce angular induction effects.

There is evidence of mutual inhibition among neurons that are "tuned" to particular spatial frequencies (K. K. DeValois \& Tootell, 1983); perhaps this interaction serves to increase selectivity of response. One might hypothesize partial or complete dominance by the neurons that are best tuned to the stimulus being displayed, in which case the $p$ curve should follow the upper envelope of receptive field responses. Since it does not (see Figure 10), additional assumptions about the nature of neural responses seem in order.

Our modeling suggests that normalizing and taking the average of the receptive fields provides a reasonably good fit to the psychological data. By normalizing, one is making the assumption that a fine line is optimal for producing induction effects, and that it produces a maximal response from large as well as small receptive fields. By averaging the normalized responses, one is adopting the concept that the perceptual judgment is based on the response from an ensemble of cells. It is likely, however, that the predicted response curves do not fully reflect the responses of the fields to all stimuli. Their responses could be shaped by cooperative interaction that serves to select the members of the set and amplify their activities. Neurophysiological research indicates that collinearity of line segments acts to synchronize the oscillations among neuronal populations (Eckhorn et al., 1988; Engel, Konig, Kreiter, \& Singer, 1991; Gray, Konig, Engel, \& Singer, 1989; Lowell \& Singer, 1992). It is possible that the perception of alignment is mediated by the relative coherence of these oscillations, thus providing a substrate for completing disconnected portions of a contour. This process might also contribute to angular induction if the 
strength of the effect was determined by the level of synchrony. We hypothesize that these interactions select and boost the activity of those neurons whose fields are centered and collinear to the tip of the test segment.

Paradiso (1988) and Vogels (1990) have argued that judgment of the orientation of a stimulus is mediated by the average of a population of cells rather than by the activity of just a few. Similar views have been advanced with respect to control of the motor system (Georgopoulos, Schwartz, \& Kettner, 1986; Lee, Rohrer, \& Sparks, 1988; Van Gisbergen, Van Opstal, \& Tax, 1987). In describing these concepts, it has been common to use the terms population coding and ensemble interchangeably, but we perceive some important differences in what is implied by these terms. The population coding models have been advanced to deal with the fact that single-cell responses are generally less precise than are the judgments and motor responses that can be rendered by an observer. By averaging the response over a pool of cells, one takes advantage of the fact that the standard error of the mean is substantially smaller than the standard deviation of the individual values; thus, the population response has the potential to provide more exact information.

Ensemble encoding, on the other hand, generally relates to the process by which various attributes of the stimulus are differentially summarized and transmitted to other parts of the nervous system. Barlow (1961) has emphasized the need to remove redundancies from the sensory information, and for this purpose spatial frequency channels appear to have special benefit (Field \& Tolhurst, 1986; Kulikowski \& Bishop, 1981a, 1981b). However, some aspects of perceptual analysis require precise spatial localization. Daugman (1985), Bossomaier and Snyder (1986), and Field (1987), among others, have argued that the Gabor function provides an effective means for accomplishing both goals, or, at the very least, is an efficient compromise.

Although Field (1987) discusses the need for interaction between orthogonal phase-selective sensors, the nature of cooperative and competitive interactions is poorly understood. It seems premature, therefore, to speculate about the broad implications of the present findings. At most, we might say that the results are consistent with the view that a scattered array of points may selectively activate an ensemble of cells whose fields vary in size by octave multiples. Further, there may be cooperative boosting of activity of fields that are collinear, and this combined activity may provide the perceptual bias that we have referred to as angular induction.

\section{REFERENCES}

Andrews, B. W., \& Pollen, D. A. (1979). Relationship between spatial frequency selectivity and receptive field profile of simple cells. Journal of Physiology, 287, 163-176.

ANDrews, D. P. (1967). Perception of contour orientation in the central fovea: Part 1. Short lines. Vision Research, 7, 975-997.

Barlow, H. B. (1961). The coding of sensory messages. In W. H. Thorpe \& O. L. Zangwill (Eds.), Current problems in animal behavior (pp. 331-360). Cambridge: Cambridge University Press.
Barlow, H. B. (1981). The Ferrier lecture. Proceedings of the Royal Society of London: Series B, 212, 1-34.

Bossomaier, T., \& SNYDER, A. W. (1986). Why spatial frequency processing in the visual cortex? Vision Research, 26, 1307-1309.

Bouma, H., \& ANDRIESSEN, J. J. (1970). Induced changes in the perceived orientation of line segments. Vision Research, 10, 333-349.

Carandini, M., \& Heeger, D. J. (1994). Summation and division by neurons in primate visual cortex. Science, 264, 1333-1336.

Carpenter, R. H. S., \& Blakemore, C. (1973). Interactions between orientations in human vision. Experimental Brain Research, 16, 287-303.

DAUGMAN, J. G. (1985). Uncertainty relation for resolution in space, spatial frequency, and orientation optimized by two-dimensional visual cortical filters. Journal of the Optical Society of America A, 2, $1160-1169$.

De VAlois, K. K., \& Tootell, R. B. H. (1983). Spatial-frequency specific inhibition in cat striate cortex cells. Journal of Physiology, 336, 359-376.

De Valois, R. L., Al. Brecht, D. G., \& Thorell, L. G. (1982). Spatial frequency selectivity of cells in macaque visual cortex. Vision Research, 22, 545-559.

Eckhorn, R., Bauer, R., Jordan, W., Brosch, M., Kruse, W., MunK, M., \& ReItboeck, H. J. (1988). Coherent oscillations: A mechanism of feature linking in the visual cortex? Biological Cybernetics, 60, 121-130.

Engel, A. K., Konig, P., Kreiter, A. K., \& Singer, W. (1991). Interhemispheric synchronization of oscillatory neuronal responses in cat visual cortex. Science, 252, 1177-1179.

FIELD, D. J. (1987). Relations between the statistics of natural images and the response properties of cortical cells. Journal of the Optical Society of America A, 4, 2379-2394

Field, D. J., \& TolHurst, D. J. (1986). The structure and symmetry of simple-cell receptive-field profiles in the cat's visual cortex. Proceedings of the Royal Society of London, 228, 379-400.

Georgopoulos, A. P., Schwartz, A. B., \& Ketrner, R. E. (1986). Neuronal population coding of movement direction. Science, 233, 1416-1419.

GiBson, J. J. (1950). The perception of the visual world. Cambridge, MA: Riverside Press.

Gray, C. M., Konig, P., ENgel, A. K., \& Singer, W. (1989). Oscillatory responses in cat visual cortex exhibit intercolumnar synchronization which reflects global stimulus properties. Nature, 338, 334-337.

GREeNe, E. (1993a). Angular induction is modulated by the orientation of the test segment but not its length. Perception \& Psychophysics, 54, 640-648.

GREENE, E. (1993b). The influence of various response factors on the amplitude of angular induction. Perceptual \& Motor Skills, 76, 323335.

Greene, E. (1994). Collinearity judgment as a function of induction angle. Perceptual \& Motor Skills, 78, 655-674.

Greene, E., \& Pavlov, G. (1989). Angular induction as a function of contact and target orientation. Perception, 18, 143-154.

Helmholtz, H. L. F. von (1962). Physiological optics: Vol. 3 (J. P. C. Southall, Trans.). New York: Dover. (Original work published 1909)

Hubel, D. H., \& WieSEL, T. N. (1962). Receptive fields, binocular interaction and functional architecture in the cat visual cortex. Journal of Physiology, 160, 106-154.

Jones, J. P., \& Palmer, L. A. (1987a). An evaluation of the twodimensional Gabor filter model of simple receptive fields in cat striate cortex. Journal of Neurophysiology, 58, 1233-1258.

JoNES, J. P., \& PALMER, L. A. (1987b). The two-dimensional spatial structure of simple receptive fields in cat striate cortex. Journal of Neurophysiology, 58, 1187-1211.

KulikowsKI, J. J., \& BISHOP, P. O. (1981a). Fourier analysis and spatial representation in the cat visual cortex. Experimentia, 37, 160-163.

Kulikowski, J. J., \& Bishop, P. O. (1981b). Linear analysis of the response of simple cells in the cat visual cortex. Experimental Brain Research, 44, 386-400.

Kulikowski, J. J., Marcelja, S., \& Bishop, P. O. (1982). Theory of spatial position and spatial frequency relations in the receptive 
fields of simple cells in cat visual cortex. Biological Cybernetics, 43, 187-198.

LeE, C., Rohrer, W. H., \& Sparks, D. L. (1988). Population coding of saccadic eye movements in neurons in the superior colliculus. $\mathrm{Na}$ ture, 332, 357-360.

LOWELL, S., \& SINGER, W. (1992). Selection of intrinsic horizontal connections in the visual cortex by correlated neuronal activity. Science, 255, 209-212.

MALLAT, S. G. (1989). A theory for multiresolution signal decomposition: The wavelet representation. IEEE Transactions on Pattern Analysis \& Machine Intelligence, 11, 674-693.

MARCELJA, S. (1980). Mathematical description of the responses of simple cortical cells. Journal of the Optical Society of America A, 70, 1297-1300.

Movshon, J. A., Thompson, I. D., \& Tolhurst, D. J. (1978). Spatial summation in the receptive field of simple cells in the cat's striate cortex. Journal of Physiology, 283, 53-77.

PARADISO, M. A. (1988). A theory for the use of visual orientation information which exploits the columnar structure of striate cortex. Biological Cybernetics, 58, 35-49.

SAKITT, B., \& BarLOW, H. B. (1982). A model for the economical encoding of the visual image in cerebral cortex. Biological Cybernetics, 43, 97-108.

TrTCHENER, E. B. (1915). A beginner's psychology. New York: Macmillan.

TOLHURST, D. J., \& DEAN, A. F. (1987). Spatial summation by simple cells in the striate cortex of the cat. Experimental Brain Research, 66, 607-620.

VAN Gisbergen, J. A. M., Van Opstal, A. J., \& TaX, A. A. M. (1987). Collicular ensemble coding of saccades based on vector summation. Neuroscience, 21, 541-555.

VoGELS, R. (1990). Population coding of stimulus orientation by striate cortical cells. Biological Cybernetics, 64, 25-31.

WATsON, A. B. (1983). Detection and recognition of simple spatial forms. In O. J. Braddick \& A. C. Slade (Eds.), Physical and biological processing of images (pp. 100-114). Berlin: Springer-Verlag.

WeinTraub, D. J., \& BRoWN, S. (1986). Perceptual plane geometry: Collinearity judgments probe the perceived orientation of an angle's sides. Journal of Experimental Psychology: Human Perception \& Performance, 12, 434-444.

Weintraub, D. J., \& Krantz, D. H. (1971). The Poggendorff illusion: Amputations, rotations and other perturbations. Perception \& Psychophysics, 10, 257-264.

Wenderoth, P., Parkinson, A., \& White, D. (1979). A comparison of visual tilt illusions measured by the techniques of vertical setting, parallel matching, and dot alignment. Perception, 8, 47-57.

WundT, W. (1893). Grundzüge der physiologischen Psychologie [Fundamentals of physiological psychology]. Leipzig: Engelmann.

\section{NOTES}

1. Previous experiments (e.g., Greene \& Pavlov, 1989) have shown that the direction of the error depends upon the sign of $\theta$. With positive values of $\theta$ (used in the present study) the error tends to be clockwise when the test segment is presented in Quadrants 1 and 3, and counterclockwise when it is presented in Quadrants 2 and 4 . These directions were designated as positive, and this convention served to maintain correspondence between the signs of $\theta$ and $\Psi$.

2. This correction was applied to adjust for a source of bias that does not appear to be due to angular induction per se. Wundt (1893) first reported that one can see a Poggendorff-like illusion even where the configuration contains no parallel lines, and he attributed it to an assimilation of the segment being judged toward either the vertical or horizontal axis. This effect is likely responsible for the mean judgment error of about $3^{\circ}$ where the configuration did not contain an induction segment, and can produce an upward (or downward) bias of the entire induction curve, depending on proximity to a cardinal axis (Greene, 1994). In the present experimental situation, this factor might be incorporated into the model as a simple added constant, but we prefer to gather more information before specifying its role in the model. Thus, as was done in previous studies (Greene, 1993a, 1993b), we have normalized the curves to be zero at the $90^{\circ}$ theta angle, where induction is predicted to be nil. After estimating the value of $p$ for each level of scatter, we add back the mean value at $90^{\circ}$ so that the theoretical line functions can be compared with uncorrected data.

3 . The collapse of the induction array along the long axis yields a distribution that approximates a Gaussian and contains the same number of points that were present in the induction array itself. Because each array contains the same number of points, we require that the stimulus Gaussians be equivalent with regard to area under the curve. As indicated previously, the solid-line stimulus is also modeled as a Gaussian having $6^{\prime}$ of effective width, in that optical blurring would be expected to produce approximately $1 \mathrm{~min}$ of spread from each edge of the line.

4. Although we believe this to be a reasonable simplifying assumption, it may be said that the impact of an array on a two-dimensional Gabor function may differ somewhat from the response that is predicted in one dimension. As one moves away from the center of the field along the long axis, the width of the field becomes narrower as a function of the Gaussian envelope that describes this dimension. The excitatory and inhibitory subfields both shrink toward the axis, and for a given scatter of points they would be more equally activated. This would have the effect of providing a reduced response from the ends of the field relative to the center, and thus the net activation of the twodimensional receptive field would be slightly less than is predicted by the one-dimensional model. We would not anticipate, however, any change in the overall response profile (which is normalized), so our analysis and conclusions should be unaffected.

5. The mechanism by which neurons in the visual system become tuned for orientation selectivity is far from settled. Among the suggested alternatives is the "winner-take-all" model, in which the cell that is optimally tuned to the orientation of the stimulus becomes dominant. We use a simplification of this notion in that only the response of the dominant cell is used by the model.

6. The classical definition of contrast is based either on a luminance edge of a contour or on area contrast; the latter is highly dependent on the edges of the areas in question. Neither definition can be readily applied to our stimuli. We adopted the view that the "contrast" of our stimuli is defined by the height of the envelope Gaussian approximating the distribution of the dots. Throughout the text, the term contrast refers to this definition.

7. For the purist, it must be acknowledged that the mean of the ensemble actually plots as a sigmoid which has been considerably attenuated relative to the curvature of the individual members of the set. It is likely that this attribute would persist across all efforts to optimize the dimensions of the Gabors that form the base set, and with respect to adjustment of scaling. On the other hand, one can perceive a slight sigmoid when sighting down the plot of the $p$ values as well; therefore, what we have been describing as a linear decline in the strength of $p$ with increasing scatter of the points may be only approximately linear. The appropriate curve could well be a highly attenuated sigmoid that reflects the mean response of receptive fields that can be modeled as Gabor functions.

(Manuscript received April 15, 1993; revision accepted for publication May 18, 1994.) 